\title{
MATHEMATICAL METHODS AND MODELS FOR RADIATION CARCINOGENESIS STUDIES*
}

\author{
A. M. Kellerer \\ Institut für Medizinische Strahlenkunde der Universität Würzburg, Versbacher Straße 5, D-8700 \\ Würzburg, Federal Republic of Germany
}

(Accepted 10 December 1985)

\begin{abstract}
Research on radiation carcinogenesis requires a twofold approach. Studies of primary molecular lesions and subsequent cytogenetic changes are essential, but they cannot at present provide numerical estimates of the risk of small doses of ionizing radiations. Such estimates require extrapolations from dose, time, and age dependences of tumor rates observed in animal studies and epidemiological investigations, and they necessitate the use of statistical methods that correct for competing risks. A brief survey is given of the historical roots of such methods, of the basic concepts and quantities which are required, and of the maximum likelihood estimates which can be derived for right censored and double censored data. Non-parametric and parametric models for the analysis of tumor rates and their time and dose dependences are explained.
\end{abstract}

Key words: Radiation carcinogenesis, neutrons, radon, tumor rates, hazard functions, censored data, competing risks, maximum likelihood methods, proportional hazards model.

\section{INTRODUCTION}

RECENT years have seen basic changes in the philosophy of radiation protection. When ICRP introduced the distinction between stochastic and non-stochastic effects [1] it may have chosen somewhat inadequate and perhaps misleading terms [2]. However it did account for a real and important difference between effects which evolve presumably from damage to one single cell and effects which result from a substantial degree of cell killing in an organ. The latter effects which have been named, or misnamed, non-stochastic fit into the older system of radiation protection which was aimed, at least in principle, at the avoidance of any detriment from ionizing radiation. The former effects which are called stochastic do not fit into the system, as there is certainly no threshold dose for the production of hereditary effects and probably no threshold dose for radiation carcinogenesis. A system of avoiding radiation effects altogether is therefore impossible and a different philosophy of radiation protection had to be introduced which aims at balancing benefits and detriments.

The changing orientation of radiation protectionHarald Rossi has, not without critical reserve, spoken of an assessment system replacing the earlier limitation system [3]-has motivated and will continue to motivate

* Work supported by Euratom Contract BI6-083-D.

Abbreviations: ICRP, International Commission for Radiological Protection; $R B E$, relative biological effectiveness; $G y$, gray, special name of the unit, $\mathrm{J} / \mathrm{kg}$, of absorbed dose.

Correspondence to: Dr A. M. Kellerer at the above address. attempts to obtain numerical estimates of the probabilities of stochastic effects at small doses. ICRP and similar bodies of experts have, indeed, published risk factors for stochastic effects $[1,4,5]$. These numbers are often taken at face value and are used to convert collective doses into numbers of resulting deaths, although the risk coefficients remain tentative, and although there is rising evidence from RBE studies for neutrons that they cannot be correct both for sparsely and densely ionizing radiations, and that the quality factors for radiation protection need to be revised.

The basic program of an assessment system, however, may persist and it is likely that similar goals will be sought in other fields, such as chemical carcinogenesis. The new, ambitious aims require that radiation protection will receive better quantitative information from molecular biology and biostatistics as well as epidemiology.

One can probably not expect numerical estimates of risks for radiation carcinogenesis within the near future even from a substantially improved knowledge of the basic lesions in DNA, their repair, their promotion and their ultimate expression. The black-box approach of epidemiology and of large scale animal experiments will therefore remain a necessity, and it will have to be accompanied by the biophysicist's attempt to provide ordering schemes for the multitude of data for different radiations, at different doses, under the influence of different dose modifying factors. At the outset of this symposium it is, therefore, useful to survey basic concepts and numerical procedures which are required in radiation-carcinogenesis studies and, particularly, in attempts to quantify the effects of small doses of ionizing 
radiations and to determine the relative biological effectiveness of sparsely and densely ionizing radiations.

\section{BASIC CONCEPTS}

\subsection{The roots of actuarial statistics}

Statistical methods were initially developed for the most predictable and most error-free processes, the motion of stars, but have later found their broadest application in sciences that deal with largely unpredictable and error-prone systems, namely biology and medicine. It is therefore not entirely surprising that methods for the rigorous analysis of dose and time dependences in carcinogenesis can be traced back to the work of a man who happens to be remembered for his astronomical work and who shared with Jaques Bernoulli-the founder of the celebrated family of mathematicians - the scientific interest in the spectacular comet of 1682. Edmund Halley derived the first tables of age-dependent mortality rates from records of the city of Breslau towards the end of the seventeenth century. His tables permitted, almost a century later, the development of actuarial methods by Daniel Bernoulli [6]. When it was first recognized that vaccination could reduce or eliminate small pox, Bernoulli joined the movement to make it mandatory. To support the demand he wished to demonstrate the resultant increase of life expectancy.

It was evident that a reliable analysis could not be performed by simply adding that fraction of the population to the survivors, after the introduction of inoculation, which had formerly been killed by small pox. Mortality at the time was so high, even at young ages, that those saved from small pox were not unlikely to die soon from other causes. Bernoulli developed an approach which is usually called the Kaplan-Meier method [7] to correct for competing risks. There is no need to deal with the fairly elementary mathematics which led to Bernoulli's result in Fig. 1. The essential point is that a procedure was utilized which was based

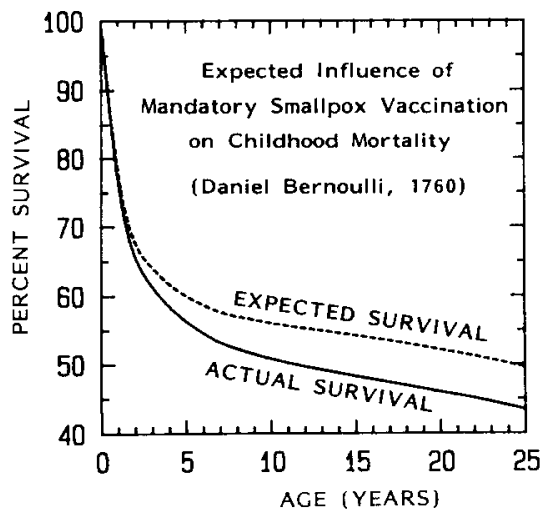

FIG. 1. Actual survival and expected survival after elimination of small pox according to the competing risk corrected calculation of Bernoulli [6]. not on the crude counting of events but on the notion of rates, or hazard rates as the statistician is now used to say. It is striking that 200 years later some important epidemiological studies in radiation carcinogenesis and some equally important large scale animal experiments are still analysed without competing risk correction.

It is of interest that there was concern even in 1760 about possible errors arising in the analysis due to hidden assumptions of statistical independence between the competing causes of death. Such doubts induced one of the referees of Bernoulli's essay-possibly d'Alembert-to block publication for more than six years.

The statistician will agree that it is not necessary to retrace Bernoulli's method. He may go further and claim, as has been done in an earlier conference on the topic [8], that mathematical methods need not be the concern of those who conduct experimental studies in radiation carcinogenesis. The only need would be to adopt mathematical notation, and the application of the well developed methods of mathematical statistics would then be routine. However, to speak a language properly, one must understand it. It is equally necessary to comprehend the terminology and recognize the essence of the models and the algorithms for which it is used.

\subsection{Basic quantities}

The fundamental quantity in studies of the age and dose dependences of stochastic events is the hazard rate. In carcinogenesis studies one may use the more specific term tumor rate. The hazard rate, $r(t)$, is the probability per unit time, at time $t$, that the event under study, e.g. the appearance of a specified tumor, takes place in an individual which is still at risk. The variable $t$ may be the age or it may be the time after a treatment whose effect is being observed. In radiation studies one may deal with additional variables such as age at exposure or dose of radiation. For brevity such additional variables will not be indicated.

While the influence of ionizing radiations on tumor rates is the primary objective, the analysis is performed in terms of certain related quantities which are less affected by statistical uncertainties and are therefore more readily estimated.

The quantity most closely linked to the tumor rate is its time integral, the cumulative tumor rate or, more generally, the cumulative hazard:

$$
R(t)=\int_{0}^{t} r(t) \mathrm{d} t .
$$

At small numerical values this quantity equals the probability for a tumor in an individual during the specified time. For less rare events the identity does not apply. The cumulative tumor rate is then larger than the probability for a tumor. It equals the expected number of events in a hypothetical individual which remains at risk even after it has been affected once or several times. In certain studies, such as the experiments on SpragueDawley rats with high incidence of mammary tumors 
which can be removed by surgery, the cumulative tumor rate is the mean number of tumors per animal (see Fig. 2 , below).

To exemplify the cumulative hazard function further, one may return to the example of mortality statistics where it is evident that at high ages the cumulative mortality rate exceeds unity. At age hundred years it is roughly 7 , if it is assumed that one out of 1000 persons lives beyond this age (see equation (4), below).

\subsection{The notion of censored data}

If no individual in a collective is removed from observation except by the events under study, one speaks of uncensored or complete data. Apart from simple survival studies one rarely deals with this ideal situation. Usually there are competing risks, such as intercurrent mortality, which remove some or most of the individuals from the population before the event of interest has taken place.

A tumor can be palpable, such as mammary tumors of the rat, or it can be rapidly lethal. In both cases it is readily discovered. If one studies such manifest tumors one has either actual times to the tumor, or-in those animals which die for unrelated reasons or are otherwise removed from the study-one has an inequality; the hypothetical time to the tumor is larger than the observed time of censoring. The combination of exact and incomplete observations is referred to by the term right censored data. For right censored data there are familiar estimates for the cumulative tumor rate or related quantities. There are also established mathematical methods for at least one type of model, the proportional hazards model (Section 3.1).

Another type of data presents more serious mathematical difficulties. If a tumor produces no appreciable life shortening, and if it can be found only incidentally in an animal dead for unrelated causes, the actual times to the tumor are never observed. If a dead animal is found to carry the tumor, the time to the tumor has been less than the time to death. If a dead animal does not carry the tumor, the hypothetical time to the tumor is larger than the observed time to death. The information is incomplete in either case, and the term double censored refers to this fact. An example of double censored data are observations of lung carcinomas in experiments of Lafuma et al. [10] on Sprague-Dawley rats. The carcinomas are found incidentally in autopsy, and there is evidence that they do not contribute substantially to life shortening.

Methods to estimate competing risk corrected incidences or cumulative hazards for double censored data have not been employed in radiation-carcinogenesis studies until recently. The same applies to models. They have not been used with double censored data. It is one of the purposes of this survey to indicate that methods exist to derive estimates of prevalences $[11,12]$ and to apply parametric or non-parametric models $[13,14]$ even with double censored data.

In fact, one needs more than methods for right censored and for double censored data, because it is often unclear whether the observation of a tumor is incidental or not. This points back to the problem of the statistical interdependence of competing risks which had arisen even in Bernoulli's initial application of actuarial statistics. Such complications are disregarded here (see e.g. $[15,16])$.

\subsection{Maximum likelihood estimates of the cumulative hazard rate or related quantities}

In a sufficiently large collective one can estimate the hazard rates directly. The study of nationwide agedependent mortality rates or tumor rates is an example. In most radiation studies numbers are too small for such a procedure. Less direct methods are then required.

The simplest method is the estimation of the cumulative tumor rate by the sum-limit estimate. The method $[17,18]$ applies to right censored data and consists in adding the reciprocals of the numbers, $N_{i}$, of individuals still at risk at all the times, $t_{i}$, when the event takes place in one individual:

$$
\hat{R}(t)=\sum_{t_{i}<t} \frac{1}{N_{i}} \pm\left(\sum_{t_{i}<t} \frac{1}{N_{i}^{2}}\right)^{0.5} .
$$

The sign ^ indicates, according to usual notation, that the quantity is an estimate, rather than the true value. The expression for the standard error is listed without derivation (see $[9,19,20]$ ). The estimate itself can be understood intuitively. If 100 individuals are still at risk the occurrence of the event in one individual amounts to an increase of the cumulative tumor rate by 0.01 . If 20 individuals are still at risk the occurrence of the event in one individual has more weight; it corresponds to an increase of the cumulative tumor rate by 0.05 . Figure 2 gives, as an example, the cumulative mammary tumor rate for the controls and the lowest neutron dose in the experiments of Shellabarger on Sprague-Dawley rats [9].

The Kaplan-Meier formula [7], also termed productlimit estimate, has been more familiar in the past. It expresses the multiplicative decrease of the 'survival probability', which is a somewhat misleading term for

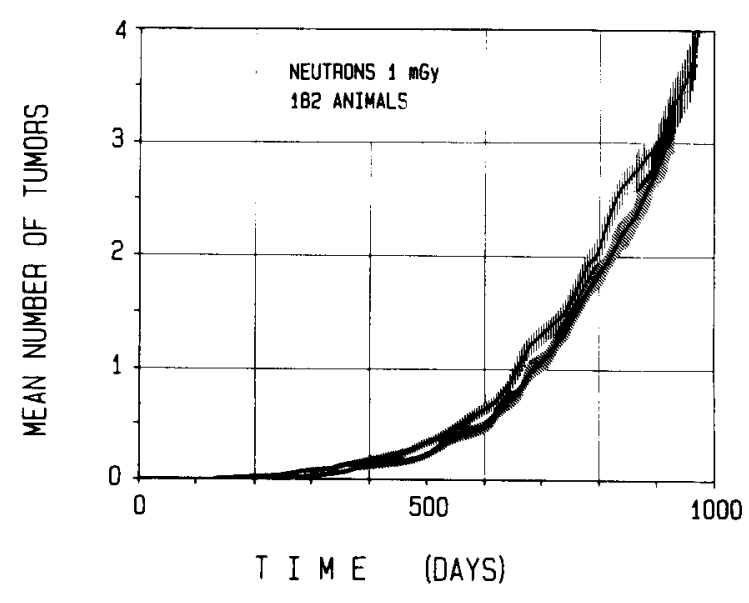

FIG. 2. The cumulative mammary tumor rate in SpragueDawley rats and its standard deviation (see equation (2)) as a function of time after exposure to $1 \mathrm{mGy}$ of $430 \mathrm{keV}$ neutrons

[9]. The lower curve gives the control incidence. 
the probability to be free from the tumor at the specified time:

$$
\hat{S}(t)=\prod_{t_{i}<t}\left(1 \frac{1}{N_{i}}\right) .
$$

Equations (2) and (3) apply when all tumor-appearance times are resolved. Evident modifications apply to grouped data.

$S(t)$ is, in the same way as the cumulative tumor rate, an abstract quantity that can not be observed directly in the presence of censoring. It is the hypothetical probability of an individual to incur no tumor, under the assumption that it is not removed from the population due to competing risks. The quantities $S(t)$ and $R(t)$ are linked by the relation:

$$
S(t)=\mathrm{e}^{-R(t)} .
$$

The considerations on models in the subsequent section will show that the hazard function, $R(t)$, is a more basic concept than $S(t)$. The utilization of $S(t)$ or $R(t)$ is, nevertheless, largely equivalent. Frequently the logarithm of $S(t)$ is plotted vs $t$ and, as shown by the example of Fig. 3, the same curves are then obtained as in a plot of $R(t)$ vs $t$.

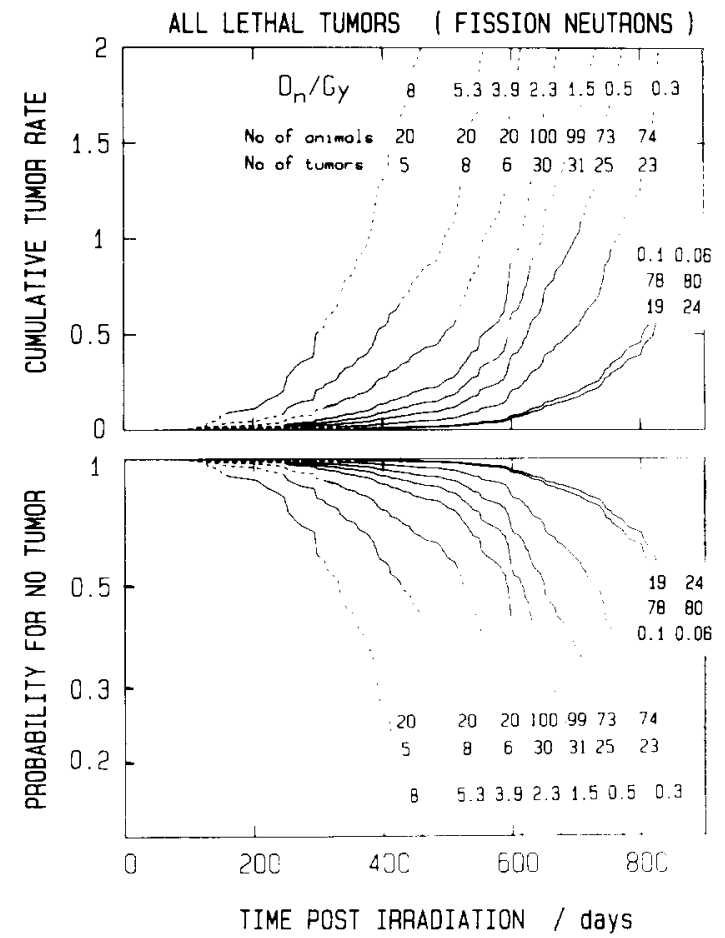

FIG. 3. The cumulative tumor rate, $R(t)$, and the probability for no tumor, $S(t)$, for all lethal tumors produced in SpragueDawley rats by the specified doses, $D_{n}$ in $\mathrm{Gy}$, of fission neutrons. The curves are maximum likelihood solutions of the proportional hazard model (see Section 3.1) for data from a current, unpublished study of Lafuma et al. The curves are given as broken lines when either less than 4 animals have already died or less than 4 animals are still alive in the group.
Equation (4) holds also for the estimates from Equations (2) and (3), provided the samples are sufficiently large. For small samples the sum-limit and the product-limit estimates need not be entirely equivalent. When the last individual in a group is affected, the Kaplan-Meier formula yields the incidence 1 ; the corresponding cumulative tumor rate is infinite. The sumlimit estimate leads, under the same condition, to more meaningful, finite values of the cumulative tumor rate which corresponds to incidences less than 1.

There are no simple formulae to estimate competing risk corrected incidences from double censored data. However, as pointed out by Hoel and Walburg [11] a considerable time ago, estimates can be derived by the method of isotonic regression which requires a relatively simple algorithm [12] but has hardly been applied to radiation carcinogenesis studies. Figure 4 gives an example of a recent application to the double censored data obtained in the study of lung carcinomas in SpragueDawley rats after neutron irradiation [14]. Although isotonic regression is a useful procedure applicable in radiation studies, its value is limited because there is no general method to obtain standard errors in small samples.

The concept of double censored data implies that the mortality rate is the same for animals with or without the tumor. It follows that the incidence, $1-S(t)$, is equal to the probability for the tumor in animals still alive at time $t$. To indicate this simple interpretation the term prevalence, rather than incidence, is used in the example of the lung carcinomas of the Sprague-Dawley rat.

\section{THE USE OF MODELS}

The direct estimates of the cumulative tumor rates or incidences represent the information from experimental data most reliably, because they are not affected by assumptions which are always inherent in models. Whenever one deals with sufficiently large experimental groups-as in the example of Fig. 2-the direct estimates are most suitable.

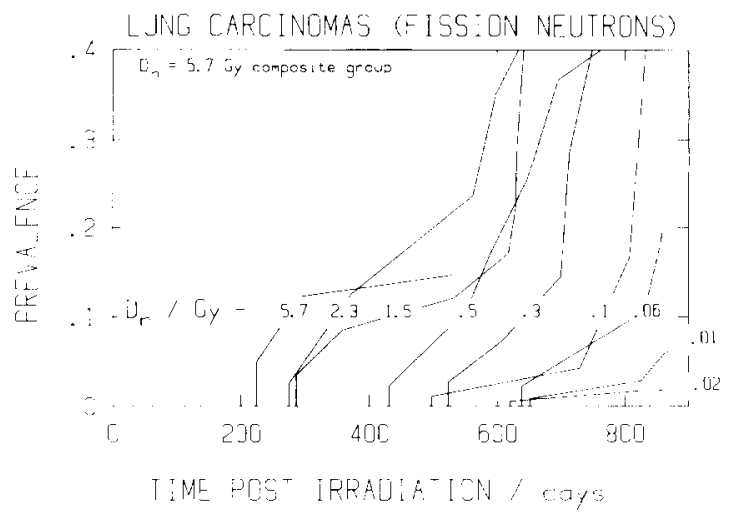

FIG. 4. Estimates of the prevalence of pulmonary carcinomas from isotonic regression applied to data of Lafuma et al. on the induction of lung carcinomas in Sprague-Dawley rats by fission neutrons [14] 
In many experimental studies, and in most epidemiological investigations, event frequencies are too small to yield useful estimates of the time dependences. Models are then required to filter out or, at least, to reduce the influences of statistical uncertainties. However, it is often difficult to separate the artefacts produced by a model from meaningful conclusions, and it is therefore necessary to compare different models in their application to a data set. The present survey is not concerned with the use of models to elucidate mechanisms. Although this aspect is always inherent in the utilization of models, the mathematical analysis is primarily directed towards the interpolation and extrapolation of data. The multitude of models and algorithms requires some guidance by means of a rough classification.

\subsection{Non-parametric models}

The term non-parametric applies to models which postulate no analytical expressions for the time or dose dependences of tumor rates. The most familiar example is the proportional hazards model. Although the approach is more general, it will be explained for the particularly simple, but not infrequent condition that one deals with a number of dose classes. The proportional hazards model admits any dependence, $R(t)$, of the cumulative tumor rate on time with the only constraint that $R(t)$ may not decrease with time. However, instead of admitting an arbitrary dependence on dose, $D$, a separation of the two variables is postulated:

$$
R(t, D)=\lambda(D) \cdot R(t) .
$$

$R(t)$ is termed the base line function, the values $\lambda(D)$ are the proportional hazards factors.

In the simple case of several dose groups $(i=1,2 \ldots$ I) one deals with one proportional hazards factor for each group:

$$
R\left(t, D_{i}\right)=\lambda_{i} \cdot R(t),(i=1,2 \ldots I) .
$$

The normalization of the proportional hazards factors is arbitrary. One can, for example, require that the geometric mean of all proportional hazards factors be unity or one can set the smallest of the proportional hazards factors equal to unity. Different normalizations amount merely to a factor in the base-line function, $R(t)$. The proportional hazards model is solved by deriving the best base-line function and the best set of proportional hazards factors in the sense of maximum likelihood. This is achieved by an algorithm due to Cox (for a FORTRAN listing see [15]). It is important to note that the base line function is not obtained from a control group, but from the entirety of all data. In fact there need not be a control group. One can, for example, utilize the proportional hazards model to compare a group of animals exposed to a neutron dose with a group exposed to a dose of X rays, and one obtains then one joint estimate of the time dependence and a ratio of hazard factors.

The proportional hazards assumption is not the only possible postulate. An alternative assumption of comparable simplicity-and more appropriate to the analy- sis of promoting factors-is the accelerated hazards model:

$$
R(t, D)=R(a(D) \cdot t)
$$

A further assumption which agrees well with the data for mammary tumors in the Sprague-Dawley rats [9] is the time-shift model:

$$
R(t, D)=R(t+s(D)) .
$$

It is evident that none of these models has full validity. However, the models are suitable to represent experimental observations within certain ranges of time and dose.

In those relatively few instances where non-parametric models have been used in radiation studies the attention has been almost exclusively focused on a proportional hazards model. The reason is not an actual preference for this model, but the attractive simplicity of the Cox algorithm which provides the numerical solutions.

Simplicity of the computational procedure is however no valid criterion for the choice of a model. Mathematical procedures for the acceleration model or the time-shift model are therefore equally necessary. More general procedures are also required because the Cox algorithm is applicable to right censored but not to double-censored data. In the recent studies on radon and neutron induced pulmonary neoplasms $[13,14]$ it has been shown that numerical solutions can be obtained for all three models and both for right-censored and double-censored data. Figure 5 gives for comparison to Fig. 4 the results obtained from the neutron-irradiation-

\section{LUNG CAACINONAS (FISSIGN NEUTBONS)}

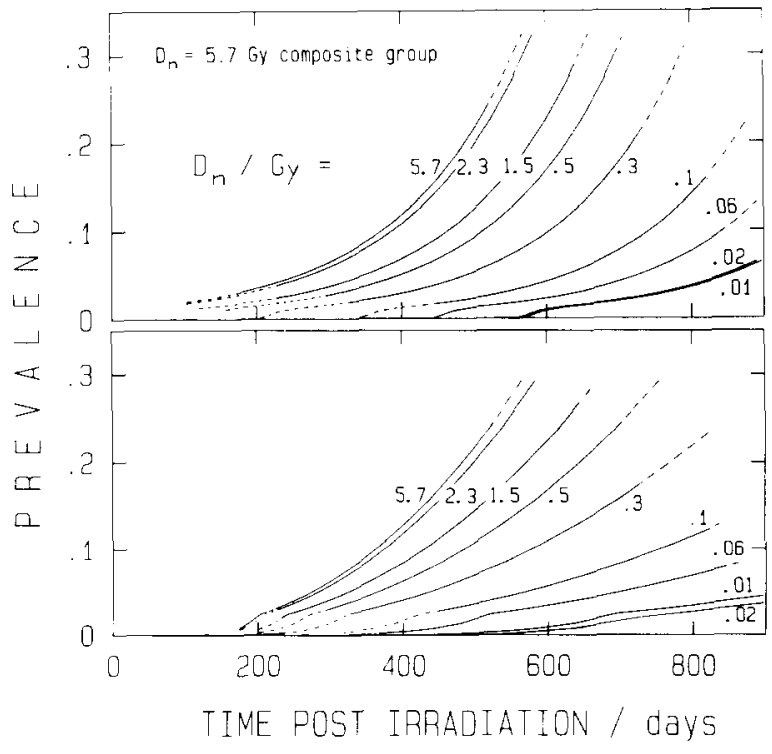

FIG. 5. Diagrams of the prevalence of lung carcinomas that correspond to Fig. 4, but are obtained as maximum likelihood solutions of the time-shift model (upper panel) or the accelerated hazards model (lower panel) [14]. 
studies with the accelerated hazards model and the timeshift model. The necessary optimization algorithms with non-linear constraints are complicated: with some data sets the convergence of the algorithms is slow or even unreliable. However these are technical difficulties which are far less demanding and costly than other aspects of epidemiological studies or large scale animal experiments. The essential point is that maximum likelihood solutions can be obtained for all three models, both for right-censored and double-censored data. The radon inhalation studies of Lafuma et al. [13] and the subsequent $\mathrm{RBE}$ analysis of neutron and radon induced lung carcinomas [14] are, up to now, the only applications. However, it is likely that the comparative utilization of non-parametric models will become common in radiation carcinogenesis studies.

\subsection{Parametric models}

Non-parametric models are attractive because they introduce a minimum of a-priori assumptions concerning the time or dose dependence of tumor rates. Additional methods are, however, required if one can not form sufficiently large dose classes to obtain meaningful results.

A partly parametric approach can be employed by postulating an analytical expression for the dose dependence. Examples are the linear-quadratic dependence or the linear-quadratic dependence modified by a reduction factor, as postulated in the mouse leukemia studies of Mole [21]:

$$
R(t, D)=\left(a D+b D^{2}\right) \mathrm{e}^{-c D} \cdot R(t) .
$$

With such models one can analyse data with varying dose values and one obtains, in addition to the nonparametric time dependence, the maximum likelihood estimates of the linear and the quadratic terms in absorbed dose which are required in biophysical considerations, in RBE studies, and in the derivation of risk coefficients.

The proportional hazards algorithm of Cox, as it is generally available [15], cannot be utilized for this modified approach. However, the necessary modifications of the numerical procedure are comparatively simple. An example for the application of the approach is the recent new analysis of the induction of osteosarcomas in the radium-224 patients of the follow-up study of Spiess [22].

In animal studies one deals frequently with dose groups but obtains event frequencies too low to permit the non-parametric approach. One can then postulate analytical expressions for the time dependence of the tumor rates. Various models have been used; however the most suitable and familiar one appears to be the Weibull model. It postulates a power function of time for the hazard rate. The simplicity of the postulated function makes it a special case both of the proportional hazards and the accelerated hazards model. With the Weibull model it is meaningless to ask whether an irradiation produces more tumors, or the same number of tumors earlier; the time dependence can be under- stood in either way:

$$
\begin{aligned}
R(t, D) & =k(D) \cdot t^{p} \\
& =(c(D) \cdot t)^{p}, \quad \text { with } k(D)=c(D)^{p} .
\end{aligned}
$$

Additional parameters, e.g. a latent period which may or may not depend on dose, have been considered. But in agreement with theory [15], it has been demonstrated [23] in an application of the Weibull model to the data of Mays et al. on plutonium induced osteosarcomas in beagles that the inclusion of the additional parameter produces no additional information and no substantially better fit to the data. The analysis of Broerse et al. of the large scale mammary tumor experiment at TNO $[24,25]$ illustrates further the application of the Weibull model.

The maximum-likelihood solutions of the parametric models present no numerical difficulties both for rightcensored and double-censored data. The equations for the likelihood that apply to the different models and to the different types of data are not given in this survey, but they can readily be derived. It is important to utilize the approach in terms of likelihood. Approximations in terms of least squares bring no advantage and can lead to faulty results when one deals with samples of small or moderate size.

\section{CONCLUSIONS}

An analysis in terms of crude incidences is still not uncommon in radiation carcinogenesis studies. Present risk estimates in radiation protection are still partly based on data which were not corrected for competing risks. This situation is rapidly changing. Numerical methods for the use of non-parametric and parametric models in radiation carcinogenesis studies will be further improved and will become generally available. This should facilitate comparisons and should make it easier to recognize artefacts introduced by models. It should also facilitate the intercomparison of results for different tumor systems and of work performed at different laboratories. Radiation is the most widely studied carcinogen, and a consistent treatment of the vast range of epidemiological and experimental data is the precondition for extrapolations required in the determination of risk coefficients and of the RBE of different types of ionizing radiations at low doses.

Important problems had to be disregarded in this survey. The difficult grey zone between right-censored and double-censored data has not been considered. Nor has it been possible to deal with the derivation of error ranges for the results obtained with parametric or nonparametric models. The necessary statistical techniques are still inadequate. But there is a growing use of bootstrap methods and related simulation procedures.

One may add the observation that tests for rightcensored data, such as the logrank test or the generalized rank-sum tests, are of comparatively recent origin. Corresponding non-parametric tests for doublecensored data do not exist, although one can construct tests in the case of large samples. 
Competing risk corrected analyses, i.e. determinations of tumor rates and their time and dose dependences, are necessary for the pragmatic purposes of radiation protection, and can further the understanding of the molecular and cellular processes underlying carcinogenesis. One may also expect that the general use of the methods will stimulate the improvement of statistical methods where they are still lacking.

\section{REFERENCES}

1. Recommendations of the International Commission on Radiological Protection (1977) Annals of the ICRP, Publication 26; Vol. 1, No. 3. Pergamon Press, Oxford, New York, Frankfurt.

2. Hulse E. V. \& Mole R. H. (1982) Reflections on the terms stochastic and non-stochastic as currently used in radiological protection. $B r . J$. Radiol. 55, 321.

3. Rossi H. H. (1984) Limitation and assessment in radiation protection and measurements, Lauriston S. Taylor Lecture No. 8. NCRP, Bethesda.

4. National Academy of Sciences, National Research Council (1980) The Effects on Populations of Exposure to Low Levels of Ionizing Radiation. Washington, D.C.

5. UNSCEAR-Report (1982) lonizing Radiation: Sources and Biological Effects. United Nations, General Assembly, 37th Session, Supplement No. 45 (A/37/45) U.N., N.Y.

6. Bernoulli D. (1766) Essai d'une nouvelle analyse de la mortalité causée par la petite vérole, et des avantages de l'inoculation pour la prévenir. Mém. Math. Phys. Acad. R. Sci. Paris, 1-45.

7. Kaplan E. L. \& Meier P. (1958) Nonparametric estimation from incomplete observations. J. Am. statist. Ass. 53, 457.

8. Groer P. G., Fry J. M., Moeschberger M. L. \& Uppuluri V. R. (1978) Environmental biological hazards and competing risks. Proc. of a Workshop, Oak Ridge, 1977. Environment Int. 1, 6 281-406. Pergamon Press, Oxford.

9. Shellabarger C. J., Chmelevsky D. \& Kellerer A. M. (1980) Induction of mammary neoplasms in the SpragueDawley rat by $430-\mathrm{keV}$ neutrons and X-rays. J. natn. Cancer Inst. 64, 821.

10. Lafuma J., Chameaud J., Perraud R., Masse R., Nenot J. C. \& Morin, M. (1976) Etude expérimentale de la comparaison de l'action toxique sur les poumons de rat du Radon 222 et de ses produits de filiation avec les émetteurs $\alpha$ la série des actinides. In Radiation Protection in Mining and Milling of Uranium and Thorium, pp. 45-53. International Labour Office, Genève.

11. Hoel D. G. \& Walburg Jr., H. E. (1972) Statistical analysis of survival experiments. J. natn. Cancer Inst. 49, 361.
12. Barlow R. E., Bartholomew D. J., Bremner J. M. \& Brunk H. D. (1972) Statistical Inference under Order Restrictions; The Theory and Application of Isotonic Regression. John Wiley, New York.

13. Chmelevsky D., Kellerer A. M., Lafuma J. \& Chameaud J. (1982) Maximum likelihood estimation of the prevalence of non-lethal neoplasms - an application to radon daughter inhalation studies. Radiat. Res. 91, 589.

14. Chmelevsky D., Kellerer A. M., Lafuma J., Morin M. \& Masse R. (1984) Comparison of the induction of pulmonary neoplasms in Sprague-Dawley rats by fission neutrons and radon daughters. Radiat. Res. 98, 519.

15. Kalbfleisch J. D. \& Prentice R. L. (1980) The Statistical Analysis of Failure Time Data. Wiley, New York.

16. Breslow N. E. \& Day N. E. (1980) Statistical Methods in Cancer Research. Vol. 1. The analysis of case-control studies. IARC Scientific Publications No. 32, Lyon.

17. Nelson W. (1972) Theory and applications of hazard plotting for censored failure data. Technometrics 14, 945.

18. Shellabarger C. J., Brown R. D., Rao A. R., Shanley J. P., Bond V. P., Kellerer A. M., Rossi H. H., Goodman L. J. \& Mills R. E. (1974) Rat mammary carcinogenesis following neutron or $\mathrm{X}$-irradiation. Symp. on the Effects of Neutron Irradiation upon Cell Function, Munich, 1973. In Biological Effects of Neutron Irradiation, pp. 391-401. IAEA-SM-179/26, Vienna.

19. Shellabarger C. J., Chmelevsky D., Kellerer A. M., Stone J. P. \& Holtzmanm S. (1982) Induction of mammary neoplasms in the ACI rat by $430 \mathrm{keV}$-neutrons, X-rays, and diethylstilbestrol. J. natn. Cancer Inst. 69, 1135.

20. Kellerer A. M. \& Chmelevsky D. (1982) Analysis of tumor rates and incidences-A survey of concepts and methods. In Proc. European Seminar on Neutron Carcinogenesis (Broerse J. J. \& Gerber G. B., Eds), pp. 209-231. EUR 8084 EN, Luxembourg.

21. Mole R. H. (1986) Radiation-induced myeloid leukemias in the mouse: experimental observations in vivo with implications for hypotheses about the basis of carcinogenesis. Leukemia Res. 10 (7), 859.

22. Chmelevsky D., Kellerer A. M., Spiess H. \& Mays C. W. (1986) A proportional hazards analysis of bone sarcoma rates in German 244-radium patients. In The Radiobiology of Radium and Thorotrast (W. Gössner et al., Eds), 3237. Urban \& Schwarzenberg, München.

23. Whittemore A. S. \& McMillan A. (1982) Osteosarcomas among beagles exposed to 239-Pu. Radiat. Res. 90, 41.

24. Broerse J. J., Hennen L. A. \& van Zwicten M. J. (1985) Radiation carcinogenesis in experimental animals and its implications for radiation protection. Int. J. Radiat. Biol. 48, 167 .

25. Broerse J. J. (1986) Dose-effect relations for mammary carcinogenesis in different rat strains, this symposium. 